\title{
Improvement of Nitrogen-phosphorus Intumescent Flame Retardant and Its Application in Polystyrene Retardant
}

\author{
Chunxue Wang ${ }^{1, a^{*}}$, Xiao Suo ${ }^{2, b}$ \\ ${ }^{1}$ School of Safety and Environmental Engineering, Capital University of Economics and Business, \\ Beijing, China \\ ${ }^{2}$ College of Resources and Safety Engineering, China University of Mining and Technology (Beijing), \\ Beijing, China \\ alittlemonkey0325@126.com, bruce88163@126.com
}

Keywords: Nitrogen - phosphorus expansion flame retardant; Silane modified; polystyrene; CFA

\begin{abstract}
With the aim of reducing thermal hazards of polystyrene (PS), the traditional nitrogen-phosphorus intumescent flame retardant ammonium polyphosphate (APP)/dipentaerythritol (DPER) was improved. Three kinds of intumescent flame retardant were made which are melamine formaldehyde resin coated APP (MFAPP)/melamine formaldehyde resin coated dipentaerythritol (MFDPER), silane-modified APP/MFDPER, and silane-modified APP/triazine charring-foaming agent (CFA) respectively. After melt blending with PS, the combustion performance of samples were studied by limiting oxygen index (LOI) and cone calorimeter (CONE). The residual carbon of samples was studied by scanning electron microscope (SEM). The results how that the silane-modified APP can improve PS retardancy, CFA can effectively improve the carbon content of PS, silane-modified APP/CFA as IFR can improve PS retardant effectively.
\end{abstract}

\section{Introduction}

Polystyrene (PS) is one of the most popular thermoplastics because it is strong functionality and has good economic returns. The fire hazard of PS is great as it is extremely flammable, and a lot of heat and smoke will be generated along with its combustion. As an environmentally friendly flame retardant, the nitrogen - phosphorus expansion flame retardant has a good application prospect[1,2,3]. Yan $\mathrm{H}[4]$ added the PPO and the APP-PER-MEL intumescent flame retardant system into the polystyrene, and found that there was a synergistic effect between the PPO and the APP-PER-MEL intumescent flame retardant system. The synergistic effect could improve the flame retardancy of PS because it improved the thermal stability effectively. Guo-sheng Liu[5] made the layered nano-silicates, metal oxides, and graphite nanosheets as the synergistic agents, and studied the synergistic agents' synergistic effect on APP/DPER system based on the substrate of polypropylene (PP). Na Wu[6] added the modified sepiolite to the PP/APP/DPER composite to study the effect of sepiolite on the retarding behavior of intumescent flame retardant PP flame. In summary, there are a few researches about the PS intumescent flame retardant. In this paper, in order to provide a reliable basis about the application of intumescent flame retardant in PS flame retardants, the traditional nitrogen-phosphorus intumescent flame retardant APP/DPER was improved.

\section{Experiment}

The experimental materials are the type II APP, MFAPP, 3- aminopropyltrimethoxysilan, acetic acid, absolute ethanol, CFA, DPER, 4A zeolite, melamine, and formaldehyde. And the preparation methods of the experiment are shown as follows.

- The modification method of APP

The dissolved solution A is obtained by dispersing 300g APP into 552g inert solvent. Mix 8g 3aminopropyltrimethoxysilan and $52 \mathrm{~g}$ deionized water together under $50{ }^{\circ} \mathrm{C}$, and the hydrolyzed coupling agent $\mathrm{B}$ is obtained by stirring the solution for $30 \mathrm{~min}$ to make the coupling agent completely 
hydrolyzed. Put $50 \mathrm{~g}$ hydrolyzed coupling agent B into the dissolved solution A under $50^{\circ} \mathrm{C}$, and filter it after reaction for 1 hour. The silane-modified APP is obtained through drying it to constant weight under the temperature of $110^{\circ} \mathrm{C}$ for 24 hours.

- $\quad$ The preparation method of MFDPER

$5 \mathrm{~g}$ melamine and $9 \mathrm{ml}$ formaldehyde solution (37\%) are added into the $100 \mathrm{ml}$ distilled water, and the $\mathrm{pH}$ is adjusted to 8-9. Turn the temperature to $90^{\circ} \mathrm{C}$ while stirring it, and stop stirring after reaction for 30min. The melamine - formaldehyde resin prepolymer aqueous solution is obtained by formulating it into $50 \mathrm{ml}$ transparent solution. $25 \mathrm{~g}$ dipentaerythritol is uniformly dispersed in $62.5 \mathrm{ml}$ ethanol solution, and $20 \mathrm{ml}$ prepolymer aqueous solution is added into it at room temperature. The $\mathrm{pH}$ of it is adjusted to 3-4. Slowly heated it to $80{ }^{\circ} \mathrm{C}$ and keep it for 2 hours. The MFDPER is obtained through cooling the solution with stirring, suction filtering, washing and drying.

- The preparation method of PS intumescent flame retardant

The PS is heated in the internal mixer at the speed of $60 \mathrm{r} / \mathrm{min}$ under $170{ }^{\circ} \mathrm{C}$. Weight the MFAPP, the silane-modified APP, the MFDPER and the 4A zeolite in proportion, and shear mix it with the preheated PS in the internal mixer. Increase the speed to $80 \mathrm{r} / \mathrm{min}$ after $5 \mathrm{~min}$, and take the mixture out of internal mixer after $15 \mathrm{~min}$. A standard sample $(100 \mathrm{~mm} \times 100 \mathrm{~mm} \times 3 \mathrm{~mm})$ is molded by the hydraulic molding machine. A piece of standard sample $(100 \mathrm{~mm} \times 6.5 \mathrm{~mm} \times 3 \mathrm{~mm})$ is cut to measure the LOI. The formulations of IFR and nitrogen-phosphorus intumescent flame retardant polystyrene are shown in Table 1 and Table 2.

Table 1 The formulation of IFR

\begin{tabular}{cccccc}
\hline NO. & MFAPP[\%] & silane-modified APP[\%] & MFDPER[\%] & CFA[\%] & 4A zeolite[\%] \\
\hline IFR1 & 28.5 & 0 & 9.5 & 0 & 2 \\
IFR2 & 0 & 28.5 & 9.5 & 0 & 2 \\
IRF3 & 0 & 30.4 & 0 & 7.6 & 2 \\
\hline
\end{tabular}

Table 2 The formulation of nitrogen-phosphorus intumescent flame retardant polystyrene

\begin{tabular}{ccc}
\hline NO. & PS[\%] & CFA[\%] \\
\hline PS0 & 100 & 0 \\
PS1 & 60 & 40 \\
PS2 & 60 & 40 \\
PS3 & 60 & 40 \\
\hline
\end{tabular}

The testing methods of the experiment are shown as follows.

- $\quad$ The determination method of LOI

The LOI of the sample is determined by using the oxygen index detector $(\mathrm{HC}-2)$, and the method is shown in this article[7].

- $\quad$ The testing method of CONE

The combustion performance testing method are shown in these articles[8,9,10]. Use the CONE (tandon redcraft) to test it and the radiant heat flux is $35 \mathrm{~kW} / \mathrm{m}^{2}$.

- The morphology analysis of the intumescent flame retardant polystyrene's residual carbon

The structure of residual carbon is characterized by the SEM (KYKY-2800B, 30kV).

\section{Experimental Analysis}

- The analysis of LOI determination

Table 3 The result of LOI determination

\begin{tabular}{cccc}
\hline NO. & PS[\%] & IFR[\%] & LOI[\%] \\
\hline PS0 & 100 & 0 & 18.2 \\
PS1 & 60 & 40 & 26.3 \\
PS2 & 60 & 40 & 27.4 \\
PS3 & 60 & 40 & 28.1 \\
\hline
\end{tabular}

The result of LOI determination is shown in Table 3. The LOI of PS0 is 18.2, which means the PS0 is extremely flammable. The LOI of PS1, PS2, PS3 are significantly increased compared with PS0. 
The LOI of PS1 (MFAPP/MFDPER) is greater than the LOI of PS2 (silane-modified APP/MFDPER). And the LOI of PS2 (silane-modified APP/MFDPER) is greater than the LOI of PS3 (silane-modified APP/CFA).

- The analysis of heat release rate

The heat release rate (HRR) profile of the samples is shown in Fig. 1. The peak of HRR (pkHRR) of PS0 is greater than other sets of samples, the pkHRR of PS0-PS3 are $922.102 \mathrm{~kW} / \mathrm{m}^{2}, 343.461$ $\mathrm{kW} / \mathrm{m}^{2}, 313.103 \mathrm{~kW} / \mathrm{m}^{2}$, and $198.536 \mathrm{~kW} / \mathrm{m}^{2}$. The decline of HRR of PS1, PS2, PS3 are relatively flat, which means that these 3 groups of flame retardants can reduce the pkHRR of PS significantly and delay the combustion of PS. The retardant effect of PS3 is the best among all the groups according to the HRR. It can be inferred that the compatibility of silane-modified APP is better than MFDPER, and the char-forming ability of CFA is better than MFDPER.

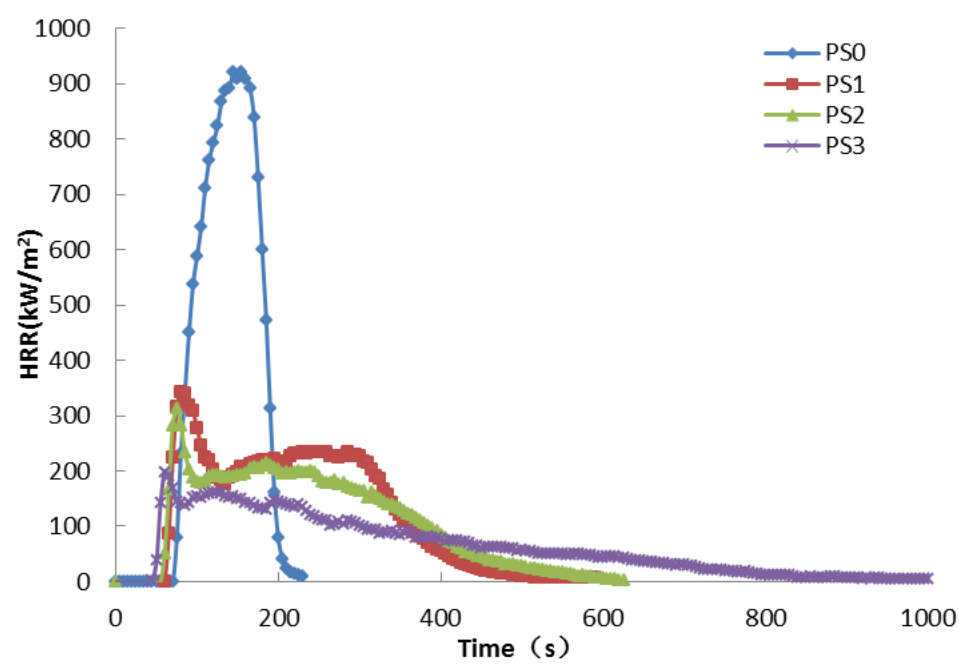

Fig. 1 The heat release rate (HRR) profile

- The analysis of smoke release rate

The smoke release rate (SRR) can be used to measure the hazard of the smoke. The profile of SRR of samples is shown in Fig. 2. The peak SRR of PS0 is $0.332 \mathrm{~m}^{2} / \mathrm{s}$, and the curve of PS0 has a sharp rise and a sharp decline. The pkSRR of PS1, PS2, PS3 are $0.135 \mathrm{~m}^{2} / \mathrm{s}, 0.103 \mathrm{~m}^{2} / \mathrm{s}$ and $0.068 \mathrm{~m}^{2} / \mathrm{s}$, the curves of them are gently and reduced significantly. According to the SRR, the PS3 has the best smoke suppression effect, and the smoke suppression effect of PS1 is the worst.

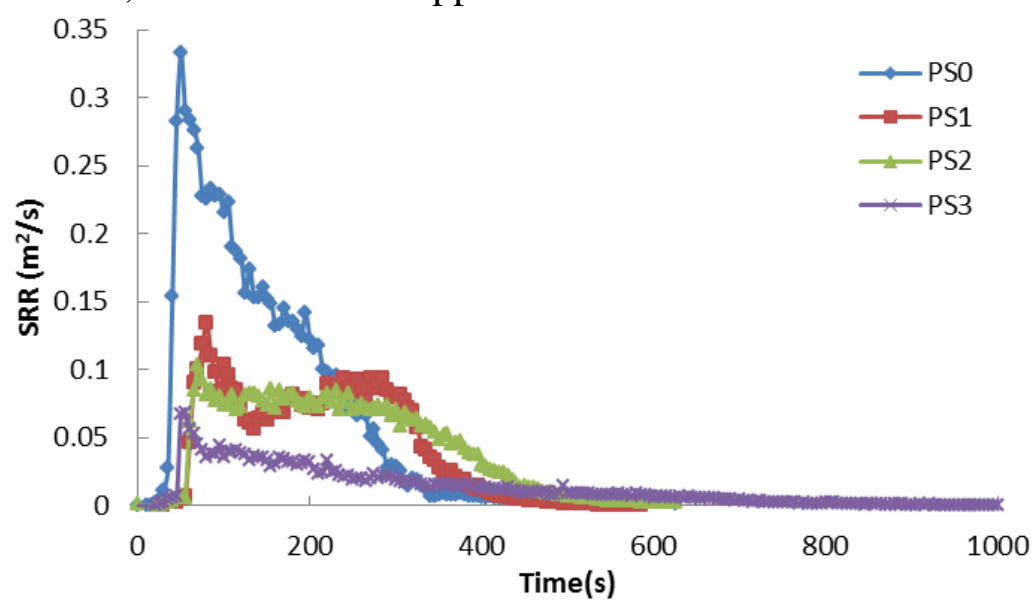

Fig. 2 The smoke release rate (SRR) profile

- The analysis of CO release rate

The CO release rate profile of the samples is shown in Fig. 3. The peak of CO release rate of PS0 is $0.020 \mathrm{~g} / \mathrm{s}$, and the curve of PS0 has a sharp rise and a sharp decline. The CO release rate of PS1, PS2, PS3 are $0.0097 \mathrm{~g} / \mathrm{s}, 0.0090 \mathrm{~g} / \mathrm{s}$ and $0.0052 \mathrm{~g} / \mathrm{s}$, the curves of them are gently. According to the curves, the CO release rate of PS0 is the minimum, and the CO release rate of PS1 is the maximum. 


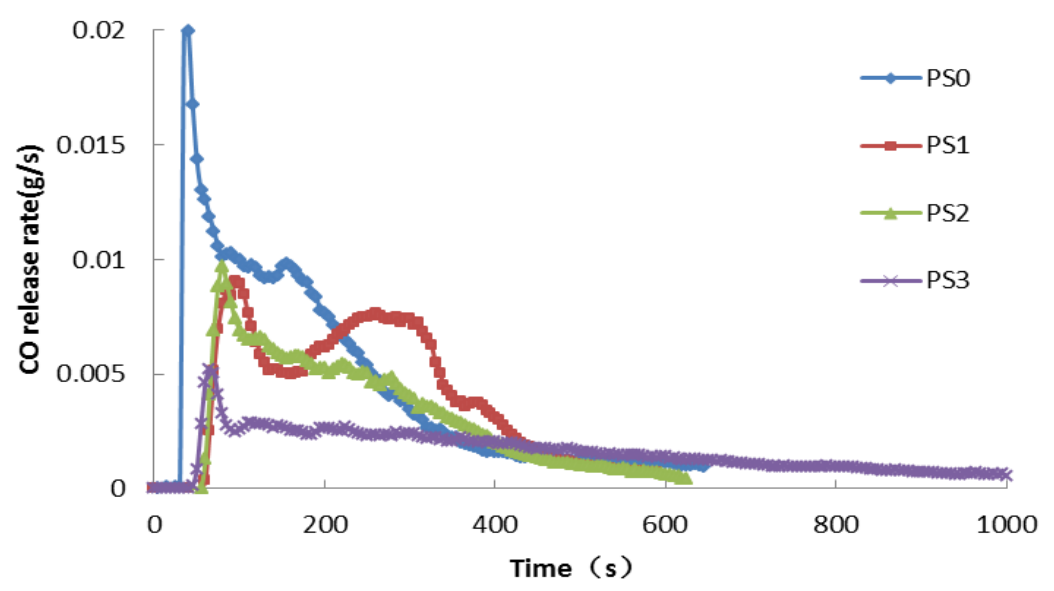

Fig. 3 The CO release rate profile

- Morphology analysis of the residual carbon

The residual carbon morphologies of PS1, PS2, PS3 are shown in Fig. 4 by scanning electron microscope (SEM), and the magnification of the SEM is 1000 times. The expansion of the residual carbon of PS1 is not obvious. The residual carbon of PS2 has a dense expansion carbon layer, but it is unevenness. The residual carbon of PS3 is uniform and dense with significant expansion. According to the morphology of the residual carbon, the char-forming ability of silane-modified APP/CFA is better than silane-modified APP/MFDPER, and the char-forming ability of silane-modified APP/MFDPER is better than silane-modified MFAPP/MFDPER.

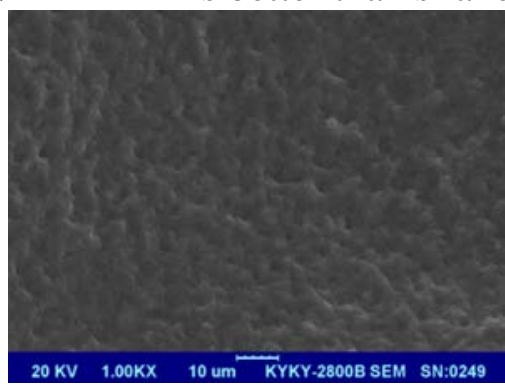

(a) PS1

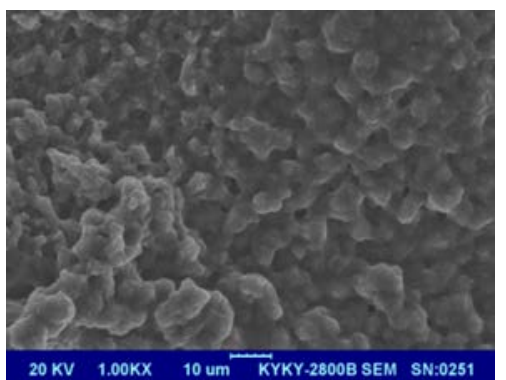

(b) PS2

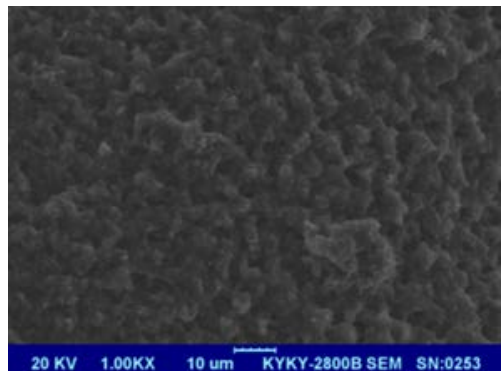

(3) PS3

Fig. 4 The residual carbon morphologies profile

\section{Summary}

Silane-modified APP/CFA with the PS substrate has the optimal flame retardant properties, silane-modified APP/MFDPER is worse than it. MFAPP/MFDPER is the worse than silane-modified APP/MFDPER. The char-forming ability of silane-modified APP/CFA is better than silane-modified APP/MFDPER, and the char-forming ability of silane-modified APP/MFDPER is better than silane-modified MFAPP/MFDPER. As the carbon source, CFA can effectively improve the a carbon content of PS. The flame retardant properties of silane-modified APP / CFA applied in the PS flame retardant as a nitrogen-phosphorus flame retardant is better than the traditional PS intumescent flame retardant.

\section{References}

[1] Cheng-ying Ma, Cui-chun Yang. Development for flame retardant of phosphorus. Modern Plastics Processing and Applications, 13. 2(2001): 38-41. (In Chinese)

[2] Hai-jun Lu, Xiao-yan Ma, Hong-xia Yan. Recent progress in phosphorus flame retardants. New Chemical materials, 29. 12(2001): 7-10. (In Chinese) 
[3] Qing-dong He, You-ming Cao, Lan Cen. Research progress on environment-friendly and high efficient intumescent flame retardants. Plastics Science and Technology, 36. 2(2008): 104-108. (In Chinese)

[4] Yan H, Dong B, Du X, et al. Flame-retardant performance of polystyrene enhanced by polyphenylene oxide and intumescent flame retardant. Polymer-Plastics Technology and Engineering, 53. 4(2014): 395-402. (In Chinese)

[5] Guo-sheng Liu. Study on synergistic intumescent flame retardant polypropylene. Beijing Institute of Technology(Doctoral Dissertation). 2014. (In Chinese)

[6] Na Wu, Chao Ding, Rong-jie Yang, et al. Effect of sepiolite on the combustion and thermal decomposition of intumescent flame retardant polypropylene. Acta Physico-Chimica Sinica, 26. 9(2010): 2429-2436. (In Chinese)

[7] Huai-li Zhang. Combustion performance test methods of plastic-the research and application of oxygen index method. Chemical Industry and Engineering, 1984: 66-71. (In Chinese)

[8] Reactiorrtcrfire tests-Heat release, smoke production and mass loss rate-Part1:Heat release rate (cone calorimeter method), ISO 566-1.

[9] Reactiorrtcrfire tests-Heat release, smoke production and mass loss rate-Part2: Smoke production rate (dynamic measurement), ISO 5660-2.

[10] Reaction-to-fire tests-Heat release, smoke production and mass loss rate-Part3: Guidance on measurement, ISO 5660-3. 